\title{
PHÂN TÍCH, THIẾT KẾ GIAO DIỆN WEBATLAS TỔNG HỢP VÙNG TÂY NGUYÊN
}

\author{
LÊ TH!̣ KIM THOA(1), NGUYẼ̃N ĐìNH KỲ(1), NGUYẼN TRƯỜNG XUÂN(2), \\ ĐINH BẢO NGỌC(2), TRỊNH VIẸT NGA(3) \\ (1) Viện Địa lý - Viện Hàn lâm Khoa học và Công nghệ Việt Nam \\ (2) Trường đai hoc Mỏ - Địa chất \\ ${ }^{(3)}$ Cục Viễn thám Quốc gia
}

\section{Tóm tắt:}

Bài báo này giới thiệu quá trình phân tích, lựa chọn ngôn ngữ HTML5, CSS3 và JQuery để thiết kế xây dựng phần giao diện cho website tổng hợp vùng Tây Nguyên cho nhóm người dùng.

\section{1. Đặt vấn đề}

Trong xây dựng một website phải thực hiện ba công việc chính là xây dựng cơ sở dữ liệu, lập trình ứng dụng website và thiết kế giao diện, không thể phủ nhận tầm quan trọng của hai việc tạo cơ sở dữ liệu và lập trình, nhưng nếu nội dung có hay nhưng phần hiển thị không đẹp, không gây ấn tượng được với người dùng, thu hút được sự chú ý của người đọc thì coi như việc xây dựng website đó đã thất bại, vì vậy việc thiết kế website là công việc hết sức quan trọng.

Một website chia làm hai phần là phần dành cho người dùng và phần quản trị website, phần dành cho người dùng thể hiện các chức năng, dịch vụ của website WebAtlas điện tử cung cấp cho người dùng do đó việc thiết kế giao diện phải hợp lý, thu hút được người sử dụng, làm cho người sử dụng dễ dàng tìm kiếm, sử dụng các chức năng của website.

Giao diện trang tương tác xây dựng phải thân thiện, rõ ràng, dễ dàng phối hợp sử dụng các chức năng của kỹ thuật đa phương tiện, trang tương tác phát hành trên Internet có độ ổn định cao, được phân quyền sử dụng và tính bảo mật cao.

Bài báo này giới thiệu kết quả nghiên

Ngày nhận bài: 06/1/2016 cứu thiết kế giao diện WebAtlas tổng hợp vùng Tây Nguyên thuộc đề tài "Xây dựng Cơ sở dữ liệu GIS và Atlas điện tử tổng hợp vùng Tây Nguyên", Chương trình Tây Nguyên 3.

2. Thiết kế và xây dụ̣ng giao diện WebAtlas vùng Tây Nguyên

2.1. Giới thiệu cấu trúc mô hình WebAtlas

Phần hiển thị bản đồ (Font End): chính là giao diện tương tác của WebAtlas vùng Tây Nguyên, WebAlas được xây dựng bằng bộ phần mềm ArcGIS Server. Hệ thống ArcGIS Server này tạo ra các services (dịch vụ) từ cơ sở dữ liệu địa lý tổng hợp (bao gồm cơ sở dữ liệu nền địa lý và cơ sở dữ liệu chuyên đề), mỗi một dịch vụ sẽ là một trang bản đồ chuyên đề tương ứng.

Mô hình chức năng dưới đây được nhìn nhận theo khía cạnh trực quan nhóm cộng đồng người sử dụng internet sẽ có các chức năng như trong hình. (Xem hình 1)

\subsection{Ngôn ngữ thiết kế giao diện}

Ngôn ngữ thiết kế giao diện được sử dụng là HTML5, CSS3 và JQuery đây là ba ngôn ngữ chính dùng để thiết kế xây dựng phần giao diện cho website

Ngày chấp nhận đăng: 07/3/2016 


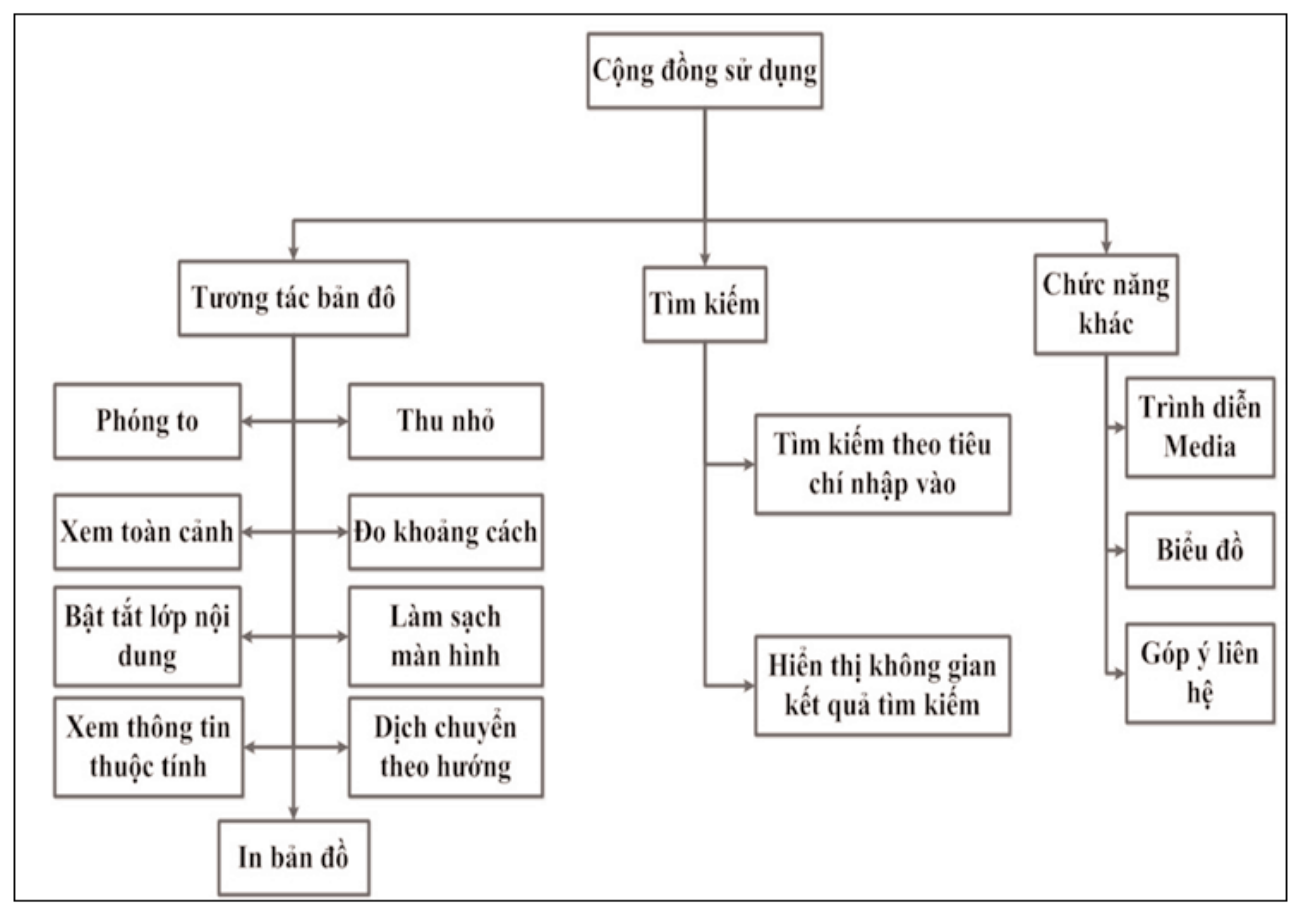

Hình 1: Biểu đồ phân rã chức năng trang quản trị

HTML (HyperText Markup Language hay ngôn ngữ đánh dấu siêu văn bản) là một ngôn ngữ đánh dấu được thiết kế để tạo nên các trang web với các mẫu thông tin được trình bày lên World Wide Web, HTML không phải là ngôn ngữ lập trình, nó là ngôn ngữ trình bày. (Xem hình 2)

CSS (Cascading Style Sheet) là các tệp tin định kiểu tầng, được dùng để miêu tả cách trình bày các tài liệu viết bằng ngôn ngữ HTML và XHTML, ngoài ra ngôn ngũ̃ này cũng dùng cho $X M L, S V G, X U L$. CSS được thiết kế chủ yếu để cho phép tách nội dung tài liệu (viết bằng ngôn ngũ $\mathrm{HTML}$ hoặc một ngôn ngữ đánh dấu tương tự) từ tài liệu trình bày, bao gồm các thành tố hiển thị (layout), màu sắc (colors), các phông chữ (fonts). Về cơ bản các trang HTML như là bộ xương, khuôn khổ cơ bản của một trang web, thì các file CSS sẽ là cụ thể hóa các thành phần của một trang nên được hiển thị như thế nào. CSS làm cho việc thiết kế và xây dựng một website trở nên dễ dàng hơn, trước khi có CSS phải sử dụng HTML để thực hiện tất cả các tiêu đề cũng như một thành phần của một trang web, CSS giúp thay đổi giao diện trang web một cách nhanh chóng và thuận tiện, ta có thể thực hiện thay đổi trên một mục nào đó rồi thực hiện trên toàn bộ trang web thay vì phải thực hiện trên từng thành phần đơn lẻ.

Các đặc điểm kỹ thuật của CSS và HTML được duy trì bởi World Wide Web Contsortium (W3C), HTML5 và CSS3 là các chương trình mới nhất cho việc thiết kế web. Các tính năng video của HTML5 cho phếp đơn giản hóa việc đưa một video vào trang web. Những ưu điểm của HTML5 và CSS3:

- Video và hiệu ứng flash: HTML5 và CSS3 cho phép người dùng xem trực tiếp video mà không cần sử dụng một plugin như Flash hoặc Silverlight. Thứ nhất, nó miễn phí không cần phần mềm Adobe Flash Plugin. Thứ hai, flash làm chậm tốc độ 


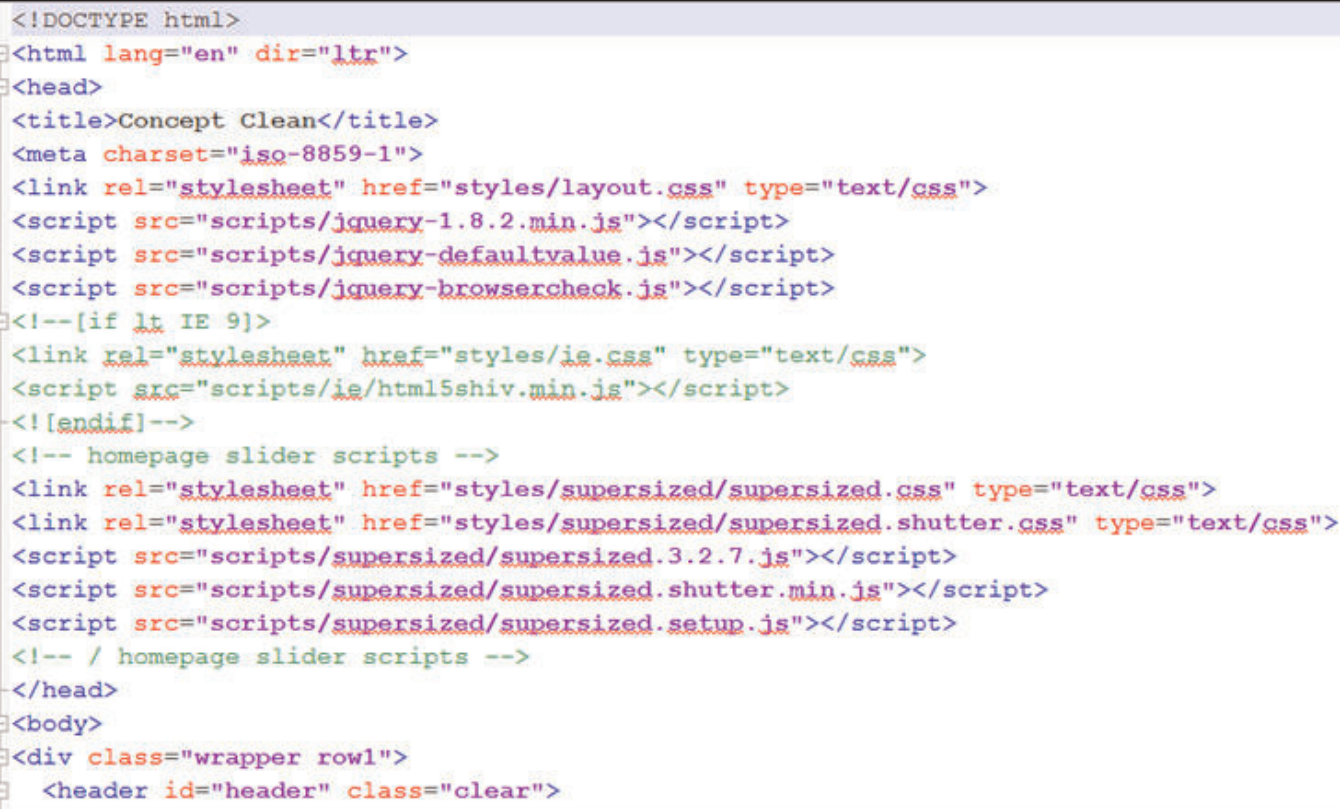

Hình 2: Ngôn ngữ HTML

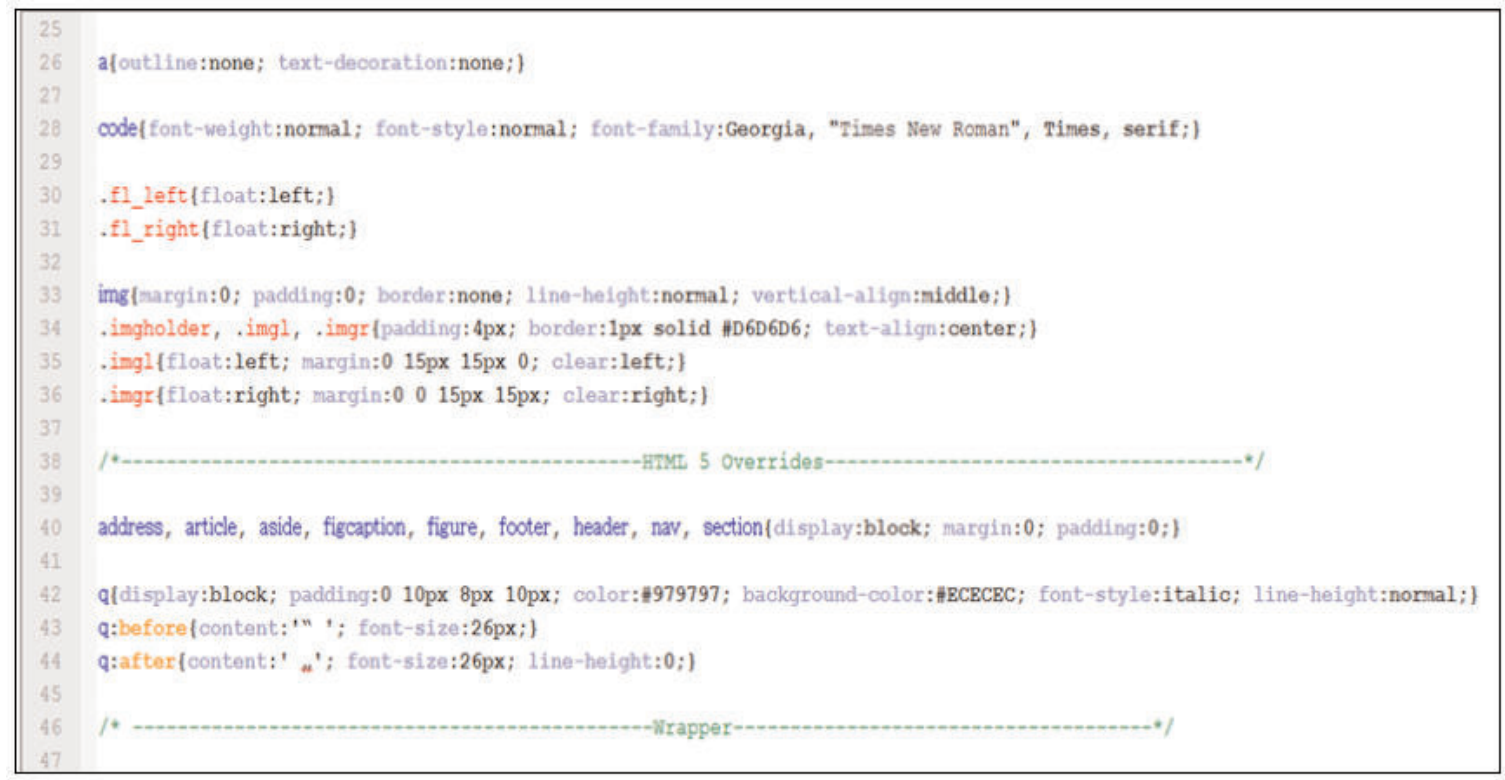

Hình 3: Ngôn ngữ CSS

duyệt website. Sau cùng, nó chỉ là một trong nhiều phần mềm đang chạy và chiếm tài nguyên hệ thống, với HTML5 tính năng video đã được xây dựng sã̃n trong nó. (Xem hinh 3)
HTML5 và CSS3 giúp các nhà thiết kế website dễ dàng hơn trong việc tạo ra các hiệu ứng động và các trò chơi tương tác mà không cần sử dụng đến Flash. 
- Kho lưu trữ offline: HTML5 cho phép các ứng dụng web có khả năng lưu trữ thông tin và tiện ích internet để sử dụng khi người dùng đang offline, điều này làm cho các ứng dụng web trở lên hữu dụng hơn. Khi đăng nhập online trở lại các ứng dụng này sẽ được load nhanh hơn. Với tính năng offline bạn có thể xác định trong trình duyệt các trang hoặc các loại trang muốn trình duyệt tiếp tục lưu trữ mà không cần phải save riêng từng trang, ta có thể thiết lập hệ thống tự động lưu một loạt trang nhất định. Điều này cũng sẽ giúp tang tốc độ load trang website khi online vì một phần của trang đã được lưu trữ trong bộ nhớ, do đó không phải chớ load lại toàn bộ dữ liệu của trang khi refesh lại.

- Công cụ thiết kế mới: HTML5 và CSS3 làm cho các ứng dụng web hấp dẫn hơn do được hỗ trợ nhiều màu sắc hơn, hỗ trợ đường cong, làm mờ và bo tròn góc. Tất cả những điều này làm cho trang web trở nên dễ nhìn và bắt mắt hơn và làm cho mọi thứ trong website trở nên sát với những gì mà nhà thiết kế website yêu cầu.

HTML5 và CSS3 được kết hợp với các công nghệ Web khác như JavaScript có thể rất hữu ích cho phong cách, định dạng và tốc độ hoạt động của bất kỳ trang web nếu được sử dụng đúng, hai ngôn ngữ sẽ cách mạng hóa cách chúng ta lập trình và xem internet với các tính năng cải tiến all-in-one, có thể loại bỏ việc phải sử dụng nhiều ứng dụng của bên thứ ba (ví dụ như Flash).

Với sự phát triển rất mau lẹ của Internet, người dùng ngày càng quan tâm hơn đến hình thức của một trang web. Trước đây một trang web chỉ cần có banner, nội dung và ít footer hời hợt là đã được cho là một trang web hoàn chỉnh. Nhưng bây giờ trang web đó phải có banner bắt mắt, nội dung hay và còn nhiều hiệu ứng lạ mắt khác nữa thì mới có thể thu hút được người đọc.

Chính vì thế những web designer bắt đầu chú ý đến các thư viện JavaScript mở như jQuery để tạo ra các hiệu ứng có thể tương tác trực tiếp với người đọc một cách nhanh chóng và dễ dàng hơn rất nhiều là sử dụng thuần JavaScript.

JQuery có cấu trúc rất mạch lạc và theo hệ thống. Cách viết code của jQuery được vay mượn từ các nguồn mà các web designer đa phần đã biết như HTML và CSS. Nếu chỉ là Designer chứ không phải coder, bạn cũng có thể dễ dàng học jQuery vì kiến thức về CSS giúp bạn rất nhiều khi bắt đầu với jQuery.

\subsection{Công cụ thiết kế giao diện Dreamweaver}

Adobe Dreamweaver CS6 là một phần mềm thiết kế web_chuyên nghiệp cung cấp giao diện hình ảnh trực quan cho việc thiết kế và chỉnh sửa các trang web HTML và ứng dụng di động. Sử dụng Fluid Grid Layout được thiết kế tương thích với đa nền tảng để tạo các layout thích ứng. Bên cạnh đó còn hỗ trợ xem lại thiết kế với Multiscreen Preview trước khi xuất bản.

\section{Một số tính năng chính của Adobe Dreamweaver:}

- Hỗ trợ jQuery Mobile, PhoneGap, CSS3/HTML5.

- Hỗ trợ xem trước thiết kế với Multiscreen Preview.

- Hỗ trợ tích hợp CMS. Bộ tích hợp Adobe Business Catalyst, Adobe Creative Suite, Adobe BrowserLab.

- Hỗ trợ FTPS, FTPES và các công nghệ hàng đầu.

- Hỗ trợ toàn diên CSS, W3C validation, hiệu ứng chuyển tiếp CSS3. Tùy chỉnh gợi ý lớp code PHP. Bộ tích hợp Edge Web Font. Tương thích với HiDPI. Truyền tải FTP hiệu quả hơn. Tìm kiếm trực tiếp trên hệ điều hành Mac $O S$. Và còn nhiều tính năng khác đang chờ bạn khám phá...(Xem hình 4) 
2.4. Giao diện tương tác bản đồ website WebAtlas điện tử

Trang tương tác WebAtlas bao gồm các thành phần sau:

- Vùng số 1: banner của website là hình ảnh thể hiện thương hiệu của website.
- Vùng số 2: thể hiện danh sách cách bản đồ chuyên đề người dùng lựa chọn để xem và tra cứu thông tin.

- Vùng số 3: hiển thị bản đồ và các công cụ tương tác tương tác với người dùng, các công cụ tương tác: (Xem hình 5)

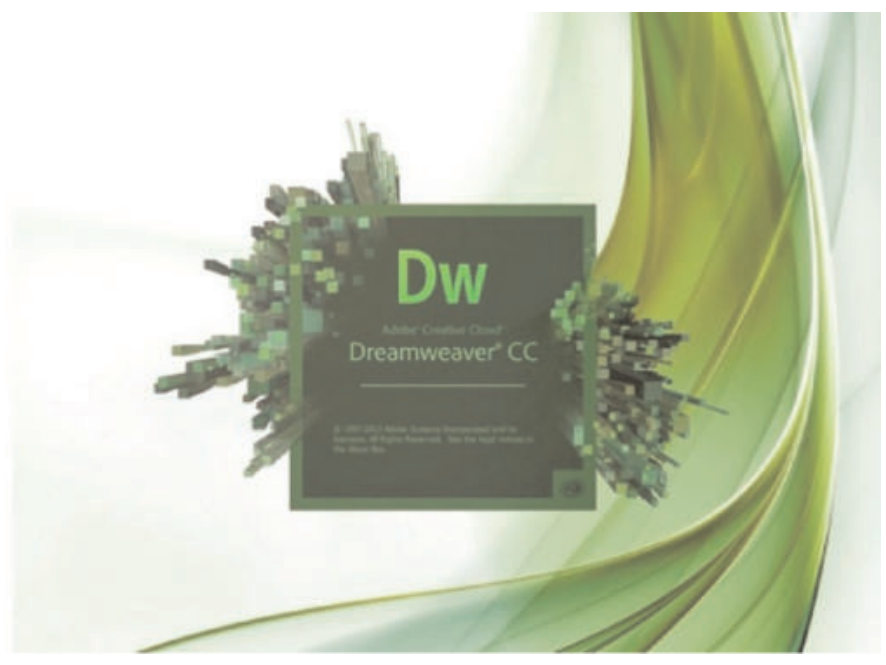

Hình 4: Adobe Dreamweaver

\section{$1: 1.014 .729$
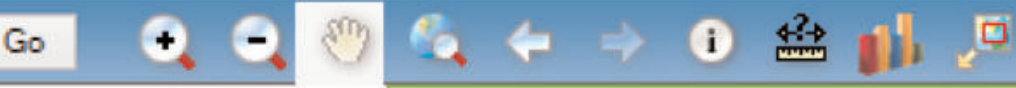

Hình 5: Giao diện các công cụ tương tác

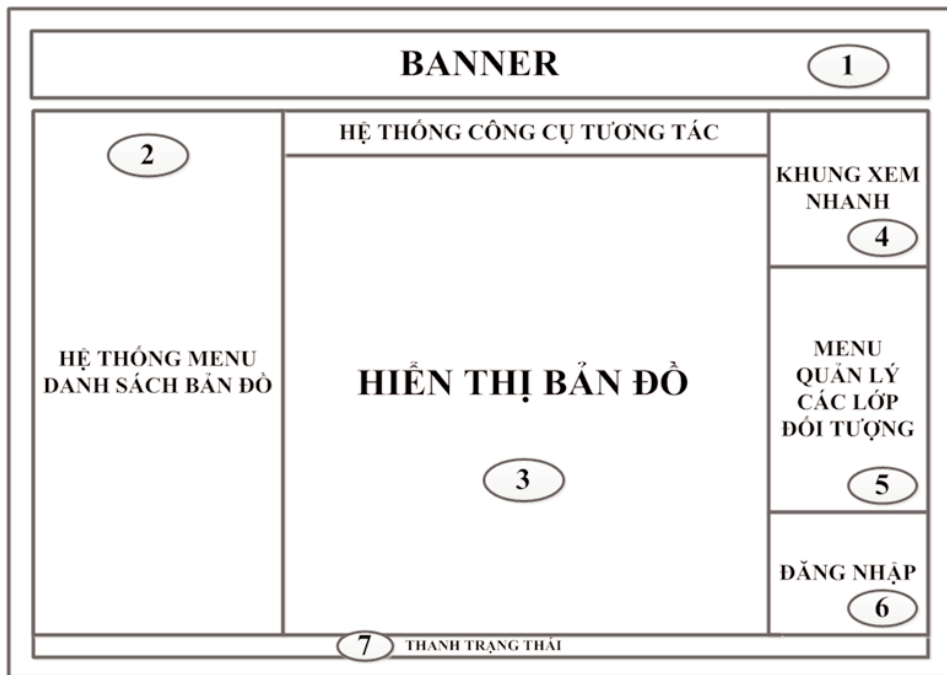

Hình 6: Giao diện tương tác WebAtlas điện tưr 
+ Công cụ phóng to: cho phép người dùng phóng to bản đồ theo phạm vi hình chữ nhật do người dùng vẽ trên bản đồ. (Xem hinh 6)

+ Công cụ thu nhỏ: cho phép người dùng thu nhỏ bản đồ theo phạm vi hình chữ nhật do người dùng vẽ trên bản đồ.

+ Công cụ đo khoảng cách: cho phép người dùng đo khoảng các giữa hai điểm trên bản đồ bằng cách dùng chuột chọn hai điểm đầu và điểm cuối cần đo khoảng cách.

+ Công cụ dịch chuyển theo hướng: đưa bản đồ hiển thị đến hướng di chuyển.

+ Công cụ xem thông tin thuộc tính: cho phép người dùng lựa chọn một đối tượng trên bản đồ bằng cách click chuột vào đối tượng đó trên bản đồ, sau đó sẽ có một khung cửa sổ hiện ra các thông tin thuộc tính của đối tượng đó.

+ Công cụ xem toàn cảnh: cho phép người dùng đưa bản đồ về tỷ lệ ban đầu như lúc mới mỏ bản đồ lên, dưa bản đồ về trạng thái tổng quát nhất.

+ Công cụ bản đồ thu nhỏ: cho phép người dùng xem bản đồ trên một cửa sổ thu nhỏ.

+ Công cụ nhập tỷ lệ bản đồ: cho phép người dùng nhập tỷ lệ bản đồ cần xem và xem bản đồ với tỷ lệ vừa nhập.

+ Công cụ in bản đồ: xuất khung nhìn hiện tại bản đồ cùng với các thông tin chú giải, khung xem nhanh (tùy theo lựa chọn của người sử dụng) ra dạng ảnh hoặc in ra giấy.

\section{Chức năng tìm kiếm gồm các chức năng sau:}

+ Tìm theo tiêu chí nhập vào: cho phép người sử dụng chọn lớp và nhập các tiêu chí cần tìm để tìm các đối tượng, kết quả tìm sẽ trả về theo danh sách được mô tả sơ lược, đồng thời cho phép người sử dụng chọn xem chi tiết từng đối tượng trong danh sách kết quả.

+ Hiển thị không gian kết quả tìm: cho phép người sử dụng xem vị trí không gian của kết quả tìm trên bản đồ.

Sử dụng thư viện Web control của ArcGIS Server để xây dựng các ứng dụng theo nhu cầu của mình. Thư viện này cung cấp các đối tượng tương tác với bản đồ (MapObject), lớp (LayerObject), điểm (PointObject), bản thân các đối tượng này đã được cài đặt các thuộc tính và các hàm cơ bản.

\subsection{Giao diện tương tác các chức} năng khác WebAtlas điện tử

\section{(Xem hinh 7)}

Các thành phần trong trang:

Vùng 1: banner là hình ảnh thể hiện thương hiệu và quản cáo cho website.

Vùng 2: hiển thị danh sách các bản đồ cần tương tác, khi người dùng chọn một bản đồ trong danh sách sẽ được chuyển đến trang giao diện tương tác bản đồ.

Vùng 3: hiển thị nội dung của media, hình ảnh, các bài viết tùy theo sự lựa chọn của người dùng.

Vùng 4: hiển thị danh sách các media (danh sách các âm thanh, video), hình rảnh của website.

Vùng 5: hiển thị danh sách các bài viết, các bài giới thiệu trong website.

Vùng 6: chức năng đăng nhập dành cho các quản trị viên. (Xem hình 8)

\section{Kết luận}

Việc tương tác với một website là rất phức tạp đặc biệt với website Atlas điện tử do website dạng này phải thể hiện khối lượng dữ liệu không gian, dữ liệu thuộc tính lớn, nhiều dịch vụ website với những định dạng dịch vụ khác nhau, vì vậy việc thiết kế giao diện cho trang tương tác phải bố trí 
Nghiên cưu - Úng dụng

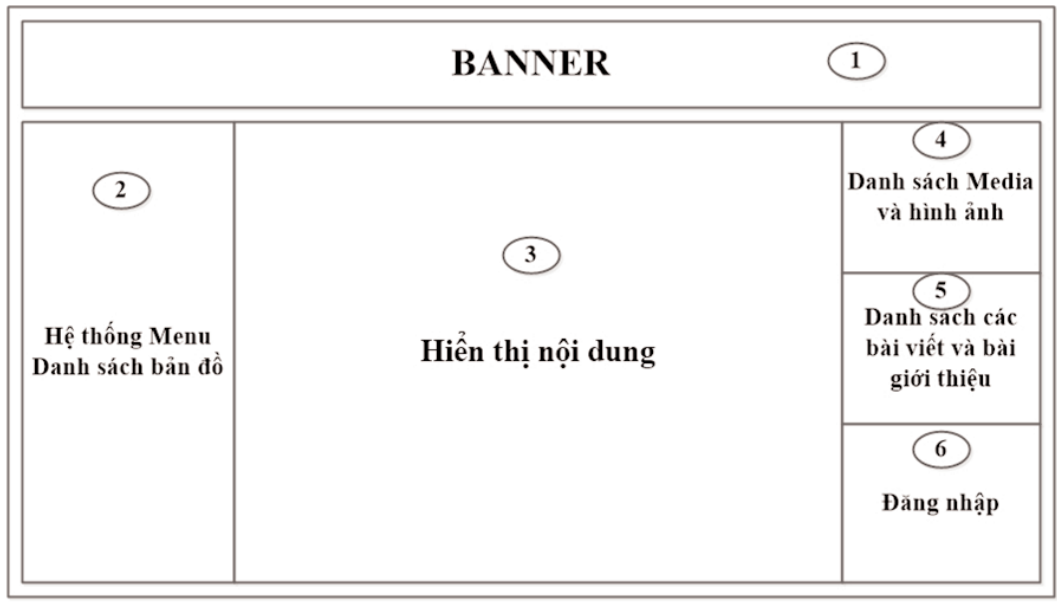

Hình 7: Giao diện tương tác các chức năng khác WebAtlas

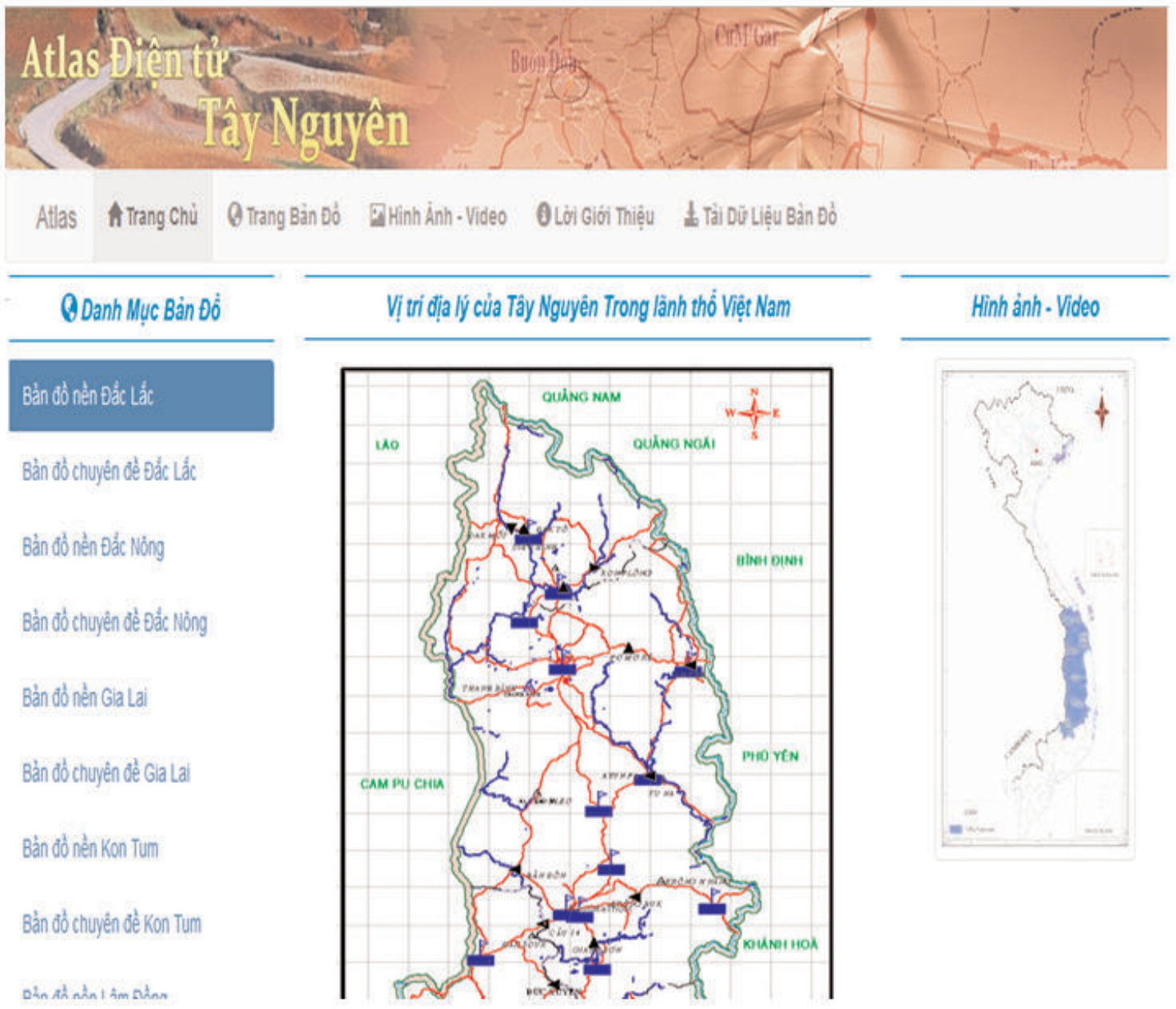

Hình 8: Trang chủ WebAtlas Tây Nguyên 
hợp lý, giúp người sử dụng dễ dàng tìm kiếm các nội dung mà mình quan tâm, ngoài việc xây dựng các giao diện cần thiết để tương tác website WebAtlas điện tử chúng ta cũng có thể sử dụng thêm các công cụ trong ArcGIS Server giúp đa dạng hóa môi trường tương tác cũng như tạo ra nhiều sự lựa chọn tương tác cho người sử dụng.

Thông qua việc xây dựng giao diện tương tác Website WebAtlas điện tử này người đọc có thể hiểu biết them về cá kiến thức mới, có thể làm tài liệu tham khảo cho các dự án làm WebAtlas cho các địa phương khác trong cả nước.

Với trang tương tác Website WebAtlas người dung có thể truy cập thông tin mọi lúc mọi nơi, thông tin quản trị được cập nhật một cách thuận tiện, liên tục và nhanh chóng, nhìn vào trang quản trị người dùng có thể dễ dàng tìm kiếm, cập nhật, chỉnh sửa, xóa các nội dung, các dịch vụ trên Website.O

\section{Tài liệu tham khảo}

[1]. Viện Địa lý - Viện Hàn lâm Khoa học và Công nghệ Việt Nam, đề tài "Xây dựng Cơ sở dữ liệu GIS và Atlas điện tử tổng hợp vùng Tây Nguyên", Chương trình Tây Nguyên 3, Hà Nội - 2012 - 2015.

[2]. (Tài liệu về HTML4 và HTML 5) http://www.w3schools.com/html/default.asp

[3]. (Tài liệu về CSS)

http://www.w3schools.com/css/default.asp

[4]. (Tài liệu về JQuery) http://www.w3schools.com/jquery/default.asp

[5]. (Xây dựng Web ADF for Arcgis Server) http://help.arcgis.com/en/sdk/10.0/serveradf_net/conceptualhelp/index.html

[6]. (Atlas điện tử tỉnh Đồng Nai) http://www.tnmtdongnai.gov.vn/Atlas/AtlasM ap.aspx. $O$

\section{Summary}

\section{WebAtlas Tay Nguyen interface analysis and design}

Nguyen Dinh Ky, Le Thi Kim Thoa, Institute of Geography - Vietnam Academy of Science and Technology

Nguyen Truong Xuan, Dinh Bao Ngoc, Hanoi University of Mining and Geology

Trinh Viet Nga, National Remote Sensing Department

This paper introduces the analysis and choosing language (such as HTML5, CSS3 and JQuer) process to design the interface component for WebAtlas Tay Nguyen for users. O

\section{NGHIÊN CỨU SỬ DỤNG MÔ HÌNH.......}

$$
\text { (Tiếp theo trang 14) }
$$

\section{Summary}

\section{Studies using rigorous sensor model to establish orthophoto VNREDSat-1 imagery}

Dr. Vu Van Chat, Dr. Pham Xuan Hoan, Army Catographic Department

VNREDSat-1 imagery was exploited since $05 / 2013$ focus on monitoring resources, environment and disaster to development social-economic, keeping defense-security, researcher and training. The paper will present the detail of procedure to establish orthophoto VNREDSat-1 imagery using rigorous sensor model (RSM) in ENVI software and assessment accuracy of the results. $O$ 